\title{
Circular Mil
}

National Cancer Institute

\section{Source}

National Cancer Institute. Circular Mil. NCI Thesaurus. Code C68870.

A unit of cross-sectional measurement of a round area equal to the area of a circle with a diameter of one mil, approximately 0.785398 square mils, or 506.707 square micrometers. 